\title{
Design, fabrication and testing of active carbon shell mirrors for space telescope applications
}

\author{
John Steeves ${ }^{a}$, Marie Laslandes ${ }^{a}$, Sergio Pellegrino ${ }^{a}$, David Redding ${ }^{b}$, Samuel Case \\ Bradford $^{b}$, James Kent Wallace ${ }^{b}$, Troy Barbee ${ }^{c}$ \\ ${ }^{a}$ California Institute of Technology, 1200 E. California Blvd, Pasadena, CA 91125; \\ ${ }^{b}$ Jet Propulsion Laboratory, California Institute of Technology, 4800 Oak Grove Dr, \\ Pasadena, CA 91109; \\ ${ }^{c}$ Lawrence Livermore National Laboratory, 7000 East Ave, Livermore, CA 94550
}

\begin{abstract}
A novel active mirror concept based on carbon fiber reinforced polymer (CFRP) materials is presented. A nanolaminate facesheet, active piezoelectric layer and printed electronics are implemented in order to provide the reflective surface, actuation capabilities and electrical wiring for the mirror. Mirrors of this design are extremely thin $(500-850 \mu \mathrm{m})$, lightweight $\left(\sim 2 \mathrm{~kg} / \mathrm{m}^{2}\right)$ and have large actuation capabilities $(\sim 100 \mu \mathrm{m}$ peakto-valley deformation per channel). Replication techniques along with simple bonding/transferring processes are implemented eliminating the need for grinding and polishing steps.

An outline of the overall design, component materials and fabrication processes is presented. A method to size the active layer for a given mirror design, along with simulation predictions on the correction capabilities of the mirror are also outlined. A custom metrology system used to capture the highly deformable nature of the mirrors is demonstrated along with preliminary prototype measurements.
\end{abstract}

Keywords: Active Mirrors, Space Telescopes, CFRP, Nanolaminate, Replication, Metrology

\section{INTRODUCTION \& BACKGROUND}

Optical-quality mirrors are heavy, expensive and difficult to manufacture. Traditional mirrors, such as that for the Hubble Space Telescope are made by grinding and polishing a thick slab of near-zero coefficient of thermal expansion (CTE) glass down to nanometer-level precision. Not only is this method difficult and expensive to implement but the areal density of such mirrors is extremely high. ${ }^{1}$ Advancements in lightweight mirrors such as those for the James Webb Space Telescope (JWST) have reduced this number greatly. ${ }^{2}$ However, as these mirrors must still be polished down to optical-quality tolerances, the associated cost and manufacturing complexity is still too great. Simpler shell-type mirrors constructed using replication techniques are also under development, ${ }^{3,4}$ however they often suffer from a lack of figure accuracy and surface quality.

Several efforts have also been made to incorporate some level of actuation into the mirror structure. This allows the mirror to 1) correct for manufacturing figure errors, 2) correct for any errors introduced during operation (ie. due to thermal variations) and 3) modify is figure in order to accommodate different optical prescriptions. Concepts incorporating a variety of materials and actuation schemes for both thick and shell-type mirrors have been proposed. The most advanced of these is the Actuated Hybrid Mirror (AHM) from AOA Xinetics. ${ }^{5,6}$ These structures are made from light-weighted silicon carbide with embedded piezoelectric stack actuators. Mirrors of this design have been demonstrated on large scales $(>1 \mathrm{~m})$ and down to optical-quality tolerances ( $<10 \mathrm{~nm}$ RMS). However, they are expensive to produce and their full-scale actuation range is limited as they are relatively stiff structures. Highly-deformable shell mirrors are also under development, ${ }^{7,8}$ however they are limited in aperture size and shape accuracy.

For further information contact John Steeves: jsteeves@caltech.edu 
We propose a novel method of constructing lightweight, highly active carbon shell mirrors (CSMs) using simple, low-cost and scalable manufacturing processes. Mirrors of this design integrate passive and active materials in order to create an active laminate structure. Replication techniques along with subsequent bonding processes are implemented, eliminating the need for any grinding/polishing steps. These mirrors have the potential to be used as segments of a primary aperture for the next-generation large aperture space telescopes. Such concepts include those for the Advanced Technology Large-Aperture Space Telescope ${ }^{12}$ or the concept envisioned by the Large Space Aperture study sponsored by the Keck Institute for Space Studies. ${ }^{11}$ Lightweight mirrors have been identified as a necessity for the realization of both of these concepts. Alternatively, this technology could also be used as a cost-effective solution for the emerging class of Earthobserving imagers based on small-scale platforms. ${ }^{13,14}$

The paper is structured as follows: Section 2 provides an overview of the CSM design as well as details on the various layers within the structure. Section 3 outlines a method to size the thickness of the active layer relative to the passive layers in order to maximize actuation. Section 4 is a study to predict the corrective capabilities of the mirror through simulation results. Section 5 outlines the current fabrication scheme in order to create the CSM prototypes. An outline of the custom metrology and testing procedure is presented in Section 6 and finally conclusions are made in Section 7.

\section{CSM OVERVIEW}

An exploded view of the various layers within the CSM is shown in Figure 1. The overall structure of the mirror is provided by a thin carbon fiber reinforced polymer (CFRP) substrate. A high-quality nanolaminate face-sheet is bonded to the front of the substrate in order to provide the reflective mirror surface. On the backside, a layer of active material is bonded providing a surface-parallel actuation scheme. A common ground, as well as custom patterned electrodes are incorporated on either side of the active material providing spatial control over the imposed actuation. Finally, a thin electrode routing layer is incorporated on the back of the active material in order to access each actuator. Such a scheme allows the CSM to be constructed extremely thin and thus highly flexible.

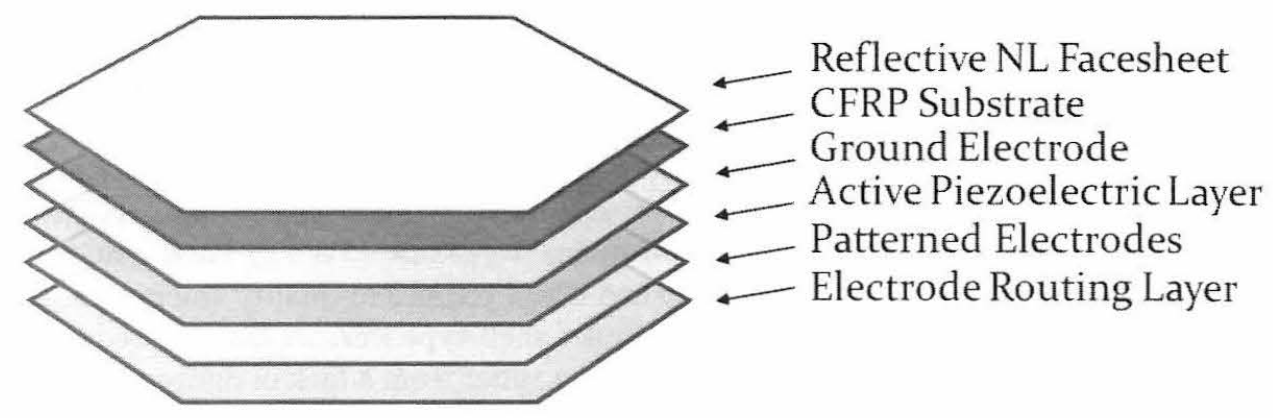

Figure 1. Exploded diagram of the CSM displaying the various layers.

\subsection{CFRP Substrate}

The mirror substrate is a composite shell made from ultra-thin plies of pre-impregnated carbon fibers and epoxy resin. Each ply of the laminate is only $30 \mu \mathrm{m}$ thick allowing quasi-isotropic laminates to be constructed while keeping the overall thickness to a minimum. For the current effort, two laminates have been considered consisting of either 8 or 16 plies. The laminate orientation is defined as $\left[0^{\circ} / 45^{\circ} /-45^{\circ} / 90^{\circ}\right]_{n s}$ where $\mathrm{n}=1$ for 8 plies and $\mathrm{n}=2$ for 16 plies ([] denotes a symmetric laminate). These CFRP shells have a total thickness of $240 \mu \mathrm{m}$ and $480 \mu \mathrm{m}$ with an areal density of $0.45 \mathrm{~kg} / \mathrm{m}^{2}$ and $0.90 \mathrm{~kg} / \mathrm{m}^{2}$ for the 8 - and 16 -ply designs respectively. 


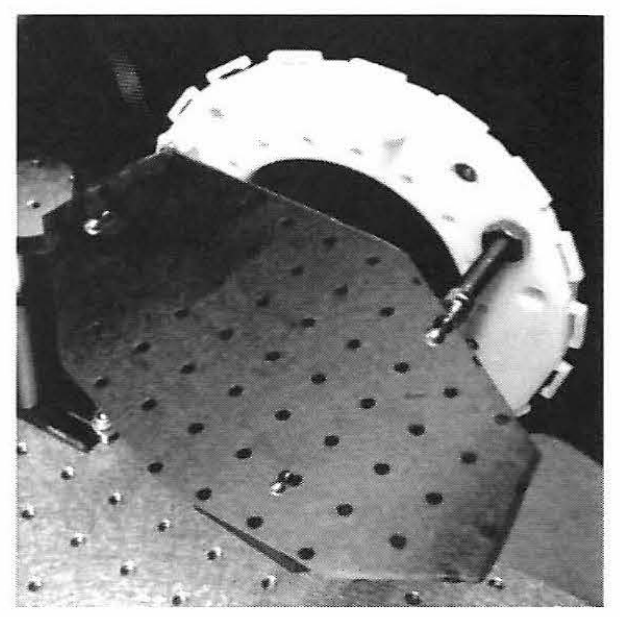

Figure 2. A cured CFRP substrate displaying smooth front surface and desired mirror figure.

Figure 2 is an example of a bare CFRP substrate. It can be seen that the as-manufactured front surface of the shell appears to be relatively smooth. However, due to thermal contraction of the epoxy during curing, the underlying unidirectional fibers protrude through the top-surface of the laminate. This effect is known as fiber print-through and is common with replicated composite optics. Figure 3(a) is a white light scanning interferometer measurement displaying this effect. It can be seen that there is significant roughness oriented perpendicular to the fiber direction.

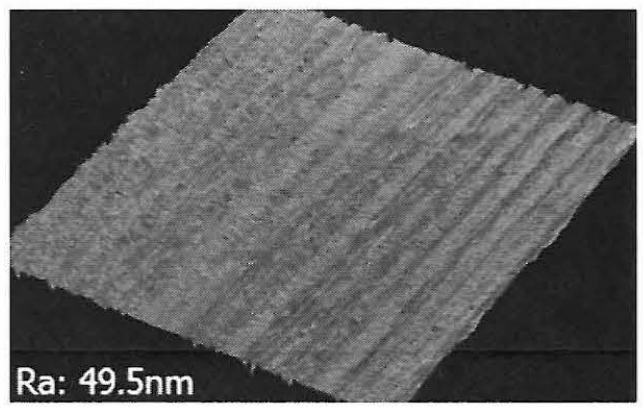

(a)

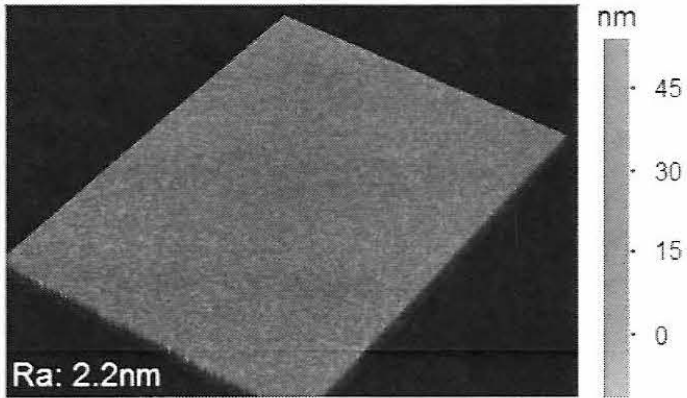

(b)

Figure 3. White light scanning interferometer (Veeco Wyko) measurements of (a) a bare CFRP substrate showing evidence of fiber print through (Ra: $49.5 \mathrm{~mm}$ ) and (b) a CSM with integrated nanolaminate facesheet showing complete elimination of fiber print-through (Ra: $2.2 \mathrm{~nm}$ ).

\subsection{Reflective Layer}

In order to obtain an optical-quality front surface, a reflective nanolaminate facesheet is bonded to the carbon substrate. Nanolaminates are multilayer metal foils formed by sputter deposition on a precision glass mandrel. ${ }^{15,16}$ The overall thickness of the nanolaminate can be tuned by varying the number of layers within the structure. Process alterations have been made in order to ensure that the nanolaminates contain an extremely low amount of internal stress. The incorporation of the nanolaminate significantly eliminates the presence of fiber print-through as shown in Figure $3(\mathrm{~b})$. 


\subsection{Active Layer}

In order to provide the surface-parallel actuation scheme, a layer of piezoelectric material is incorporated onto the backside of the CFRP substrate. The d31 piezoelectric coupling coefficient is utilized by applying an electric field through the thickness of the material resulting in a proportional in-plane strain. As the material is bonded to the CFRP substrate, this in turn produces an out-of-plane curvature change of the mirror.

Several candidate materials have been explored as options for the active layer. Due to its high actuation capabilities thin plates of Navy Type II Lead Zirconate Titanate (PZT-5A) have been implemented for the current efforts. Table 1 is a summary of the properties for this material.

Table 1. Material properties for PZT-5A. ${ }^{17}$

\begin{tabular}{lr}
\hline \hline Property & Value \\
\hline Modulus, E $(\mathrm{GPa})$ & 66.0 \\
Poisson's Ratio, $\nu$ & 0.35 \\
Piezoelectric Constant ${ }^{*}, \mathrm{~d} 31(\mathrm{pC} / \mathrm{N})$ & -250 \\
Maximum Electric Field*, $E_{\max }(\mathrm{MV} / \mathrm{m})$ & 0.8 \\
\hline *Measured value &
\end{tabular}

A continuous ground plane and patterned electrodes are printed on the top and bottom surfaces of the piezoelectric layer using silver nano-particle ink and a Dimatix 2800 Materials Printer. ${ }^{18}$ This allows high voltages to be applied to the various in-plane locations on the mirror. The pattern, shown in Figure 4 , is a custom design optimized to correct for specific modes of figure error associated with the CSM. An overview of this process can be found in Laslandes, 2014.

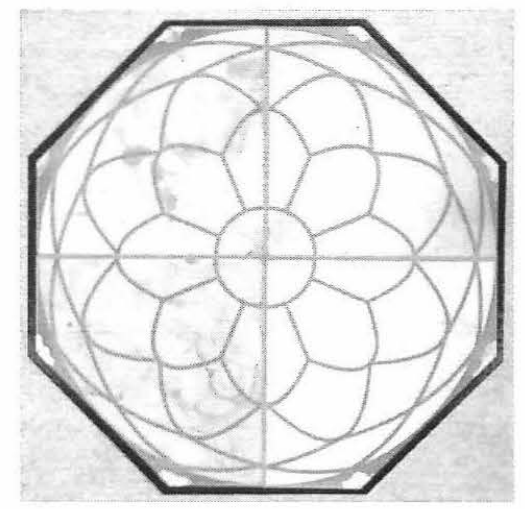

Figure 4. Backside of a $150 \mathrm{~mm}$ dia. CSM prototype containing four PZT-5A plates with a custom electrode pattern designed to correct for astigmatism-based errors.

\subsection{Electrode Routing Layer}

Due to the relatively large number of actuation channels, active mirrors often contain cluttered, bulky comnecting wires that can impart shape errors onto the mirror surface. In order to alleviate this problem for the CSMs, conductive electrode traces are printed on a thin Kapton routing laver as shown in Figure 5. Comnections to the underlying electrode pads are then made using through-thickness vias and conductive epoxy. The pattern is designed to route the traces away from the active surface of the mirror to a flexcable connector where connections to the control electronics can be made using more standard cabling techniques. The low modulus and thickness of the Kapton layer allows the CSM to remain highly flexible during actuation. 


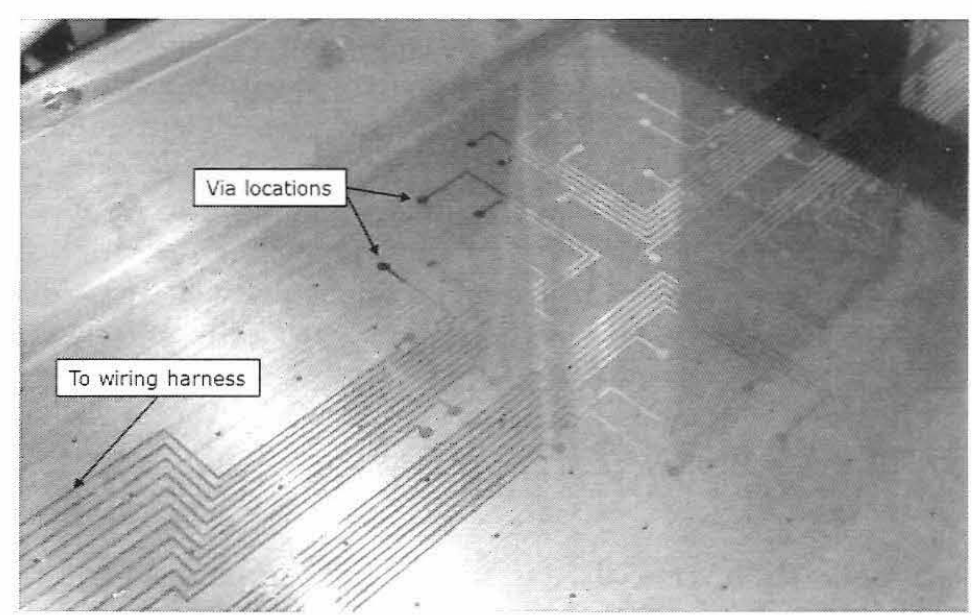

Figure 5. $25 \mu \mathrm{m}$ thick Kapton routing layer with printed electrode traces.

\section{ACTIVE LAYER DESIGN}

Since the CSMs are extremely thin structures, the relative thickness of each layer has a significant effect on the overall mechanical behavior. The thickness of the active layer is of particular importance as small changes in this value can have a large impact on the mirror's actuation capabilities. If the active layer is too thick it will dominate the flexural stiffness of the mirror while if it is too thin there will not be enough actuation authority in order to correct for the expected deformations. In order to determine the optimal thickness of the active layer a linear analysis based on Classical Lamination Theory (CLT) was performed. ${ }^{20}$ The procedure is as follows:

Given a laminate constructed from $n$ separate layers, the overall stiffness matrix can be determined relating mid-plane strains and out-of-plane curvatures, $\epsilon$ and $\kappa$, to resultant forces and moments, $\mathbf{N}$ and $\mathbf{M}$, respectively, as follows:

$$
\left[\begin{array}{l}
\mathrm{N} \\
\mathrm{M}
\end{array}\right]=\left[\begin{array}{ll}
\mathrm{A} & \mathrm{B} \\
\mathrm{B} & \mathrm{D}
\end{array}\right]\left[\begin{array}{c}
\epsilon \\
\kappa
\end{array}\right]
$$

where:

$$
\mathbf{A}=\sum_{k=1}^{n}\left(Q_{i j}\right)_{k}\left(z_{k}-z_{k-1}\right), \quad \mathbf{B}=1 / 2 \sum_{k=1}^{n}\left(Q_{i j}\right)_{k}\left(z_{k}^{2}-z_{k-1}^{2}\right), \quad \mathbf{D}=1 / 3 \sum_{k=1}^{n}\left(Q_{i j}\right)_{k}\left(z_{k}^{3}-z_{k-1}^{3}\right)
$$

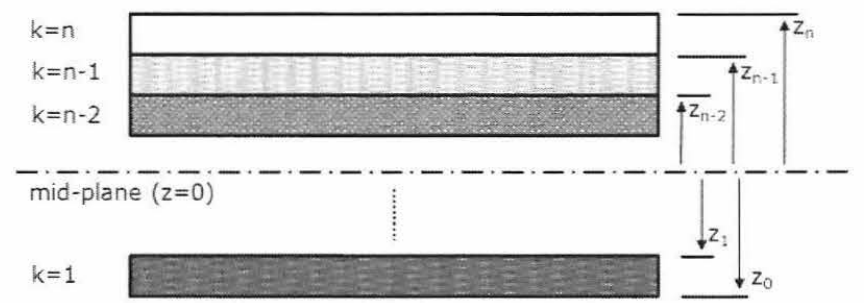

Figure 6. Through-thickness coordinate definition for the Classical Lamination Theory analysis.

where $z_{k}$ is the through-thickness coordinate of the $k^{\text {th }}$ interface and $\left(Q_{i j}\right)_{k}$ is the orthotropic stiffness matrix of the $k^{\text {th }}$ material as shown in Figure 6. However, for this study the inverse problem is of interest: given a 
set of resultant forces and moments produced by the active nature of the piezoelectric material, what are the resulting mid-plane strains, and more importantly out-of-plane curvatures of the structure? This is given as:

$$
\left[\begin{array}{c}
\epsilon \\
\kappa
\end{array}\right]=\left[\begin{array}{ll}
\mathrm{A} & \mathrm{B} \\
\mathrm{B} & \mathrm{D}
\end{array}\right]^{+}\left[\begin{array}{c}
\mathrm{N}_{\text {piezo }} \\
\mathrm{M}_{\text {piezo }}
\end{array}\right]
$$

where:

$$
\begin{aligned}
\mathbf{N}_{\text {piezo }} & =\int_{z_{a c t, b o t}}^{z_{a c t, t o p}} \mathrm{Q}_{\mathrm{act}} \mathrm{d}_{\mathrm{act}} E d z \\
\mathrm{M}_{\text {piezo }} & =\int_{z_{a c t, b o t}}^{z_{a c t, t o p}} \mathrm{Q}_{\mathrm{act}} \mathrm{d}_{\mathrm{act}} E z d z
\end{aligned}
$$

where ${ }^{+}$denotes the pseudo matrix inverse, $d_{\text {act }}$ is an array of the piezoelectric constants for the active material, $z_{a c t, t o p}$ and $z_{a c t, b o t}$ are the top and bottom coordinates of the active material and $E$ is the applied through-thickness electric field.

Table 2. CSM laminate design

\begin{tabular}{lll}
\hline \hline Layer & Material & Thickness $(\mu \mathrm{m})$ \\
\hline 1 & Nanolaminate & 50.0 \\
2 & CFRP & $240.0(8-$ ply $)$ \\
& & $480.0(16-$ ply $)$ \\
3 & PZT-5A & Variable \\
4 & Kapton & 25.0 \\
\hline
\end{tabular}

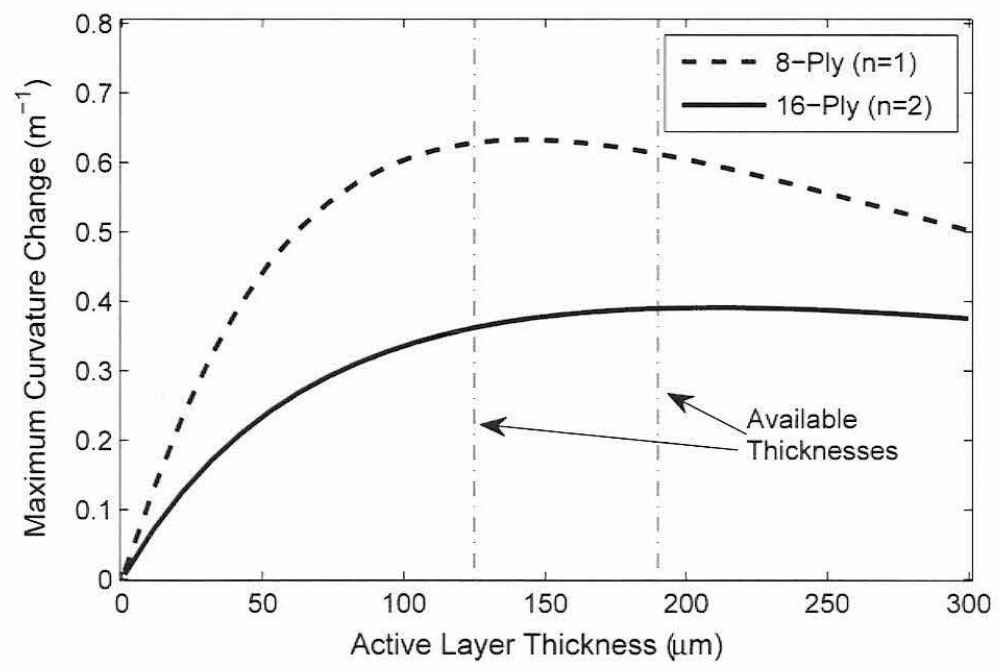

Figure 7. Maximum curvature change due to actuation as a function of active layer thickness.

Using the formulation above, the maximum curvature change of an 8-ply and 16-ply laminate was calculated as function of the active layer thickness. Table 2 outlines the various layers within the two designs. The thickness of the nanolaminate and Kapton routing layer are set to $50 \mu \mathrm{m}$ and $25 \mu \mathrm{m}$ respectively as these are the current values used in a practical setting. Figure 7 displays the results of this process. The first thing to note is that the maximum curvature change is significantly larger for the thinner 8-ply laminate. This is as expected as the flexural stiffness of the 16-ply CFRP substrate is much higher, thus providing more resistance to deformation. Next, it is evident that for both substrates and small active layer thicknesses 
there is approximately a linear increase in actuation capabilities with the active layer thickness. However, this linearity starts to diminish rapidly for active layer thicknesses over approximately $40 \mu \mathrm{m}$. For the 8-ply substrate, an optimal active layer thickness of $150 \mu \mathrm{m}$ is determined to maximize the actuation capabilities of the mirror. For the 16-ply substrate this point is shifted further to the right, having an optimal value of $240 \mu \mathrm{m}$. However it can be seen that very minimal gains are realized after a certain point $(\sim 150 \mu \mathrm{m})$. As the PZT material is the most dense component in the CSM, there is a trade between obtaining slightly higher actuation capabilities while significantly increasing the overall mass of the mirror. Therefore, taking into account practical constraints what is currently available, an active layer thickness of $125 \mu \mathrm{m}$ was chosen for both the 8-ply and 16-ply designs.

\section{SIMULATION OF CORRECTION CAPABILITIES}

In order to make predictions on the correction capabilities of various designs, the CSM was modeled using: Abaqus CAE/Standard 6.12. ${ }^{21}$ The details of this analysis can be found in Steeves, 2012. Figure 8 displays the modeled actuation pattern as well as the influence functions associated with the unique electrode positions. It is apparent that the CSM design has significant actuation capabilities as each influence function has a full-scale peak-to-valley (PV) deformation range of $45-100 \mu \mathrm{m}$ for a $16-p l y, 150 \mathrm{~mm}$ dia. design.

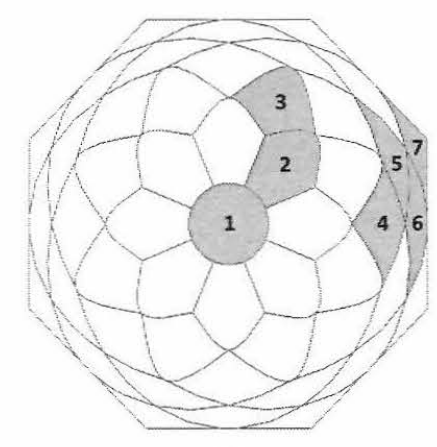

(a)
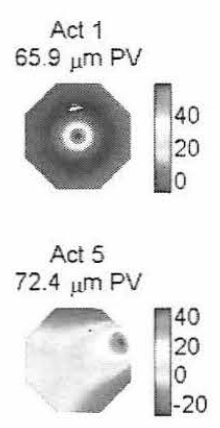
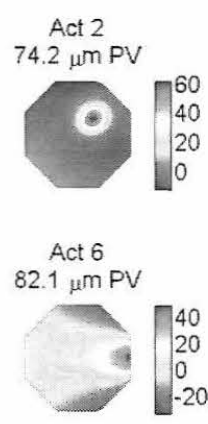
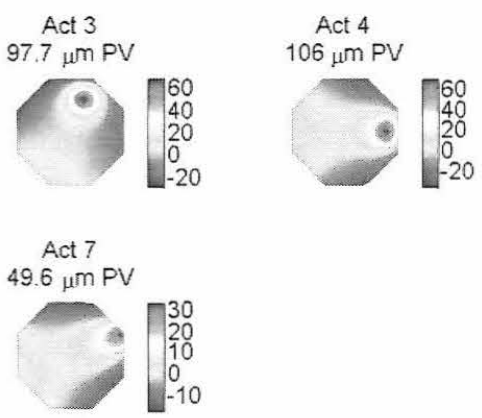

(b)

Figure 8. (a) Actuation pattern used for the CSM model showing the unique actuator locations. (b) Shape and magnitude of the corresponding influence functions for a 16-ply design.

Using the calculated influence functions a variety of performance simulations can be conducted. A constrained least-squares algorithm taking into account voltage limitations of the piezoelectric material is used in order to determine the necessary voltage map required for correction (see Laslandes, 2014 for more details on this process). Figure 9 displays the result of one such case where the active elements are used to correct a 16-ply mirror with $50 \mu \mathrm{m}$ of initial astigmatism-based error. It can be seen that with this magnitude of initial error, $0.76 \mu \mathrm{m}$ of residual error is present after correction (considering $95 \%$ of the overall aperture). It can also be seen that 12 of the 49 actuators are saturated at their maximum voltage values $( \pm 100 \mathrm{~V})$, while others are operating at much lower values. The saturated actuators are those located around the perimeter of the mirror as these have the greatest influence when performing astigmatism correction. This is also where the majority of residual error is present.

Figure 10 is a more comprehensive study detailing the performance of an 8-ply and 16-ply CSM by observing the amplitude of residual error after correction as a function of initial astigmatic error amplitude. Several observations can be made from this figure. The first is that for small initial errors $(<45 \mu$ m RMS), both the 8-ply and 16-ply designs have correction factors of approximately $99 \%$, demonstrating significant correctability. It can also be seen that the 16-ply mirror slightly outperforms the 8-ply design over this range of initial errors. This is due to the fact that the influence functions of the stiffer 16-ply mirror have a slightly more global behavior in comparison to those of the 8-ply design. This essentially acts as an inherent 


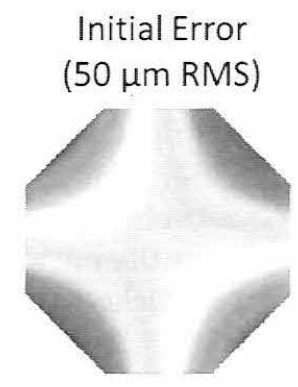

(a)

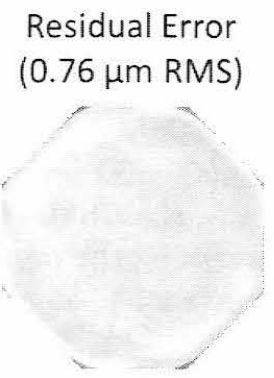

(b)
Voltage Map

( $\pm 100 \mathrm{~V}$ Limit)

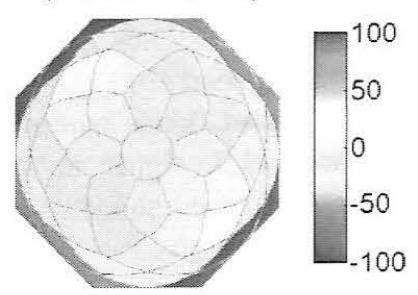

(c)

Figure 9. Simulation results for a 16-ply CSM model showing: (a) the $50 \mu \mathrm{m}$ RMS of initial astigmatic error, (b) the residual error of $0.76 \mu \mathrm{m}$ RMS after correction (considered over $95 \%$ of the pupil), and (c) the corresponding actuator voltages required for correction.

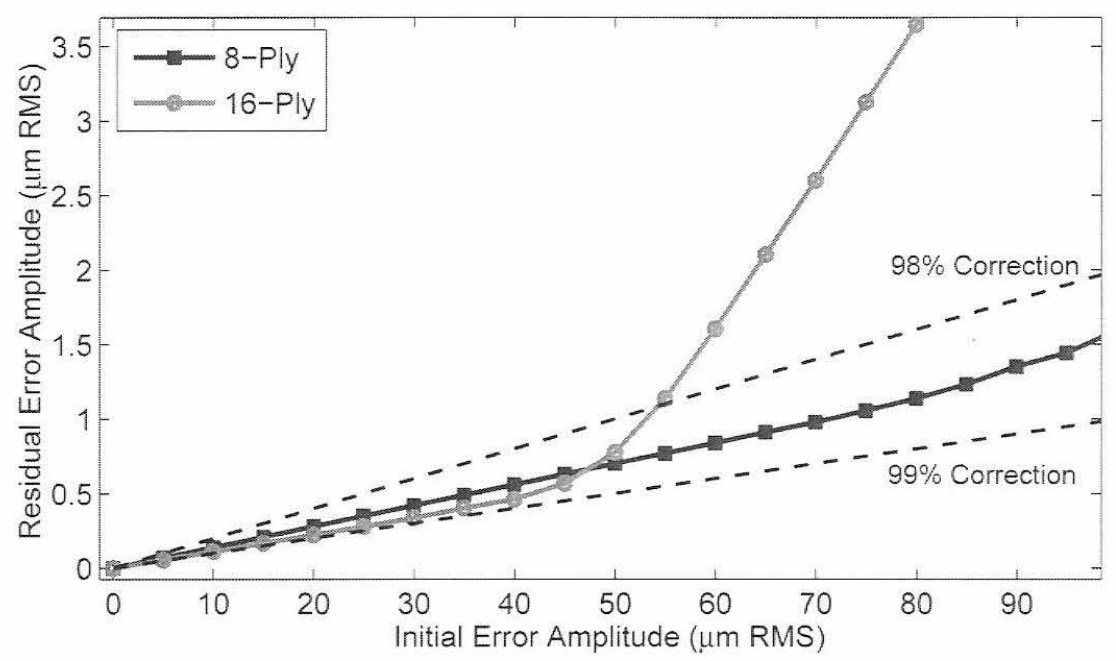

Figure 10. Comparison of the correction capabilities for a CSM constructed from 8 and 16 plies.

low-pass spatial filter, thus reducing the magnitude of residual error. The next observation is related to the effect of actuator saturation. For the 16-ply mirror, as the magnitude of initial error is increased beyond $45 \mathrm{~mm}$, the magnitude of residual error starts to increase drastically. This is due to the fact that several of the actuators have reached their voltage limit and can no longer support any further corrections. Since the 8-ply mirror is more compliant, and thus the influence functions have a higher full-scale deformation value, this effect is delayed and the mirror can correct for larger initial errors without saturation.

\section{PROTOTYPE FABRICATION}

Figure 11 is a schematic of the current fabrication process for the CSM prototypes. First, a lay-up of unidirectional carbon fiber prepreg is placed on top of a polished glass mandrel (Step 1). The laminate is vacuum bagged and autoclave cured at elevated temperature and pressure to provide consolidation of the plies. During this process the CFRP laminate replicates the surface of the underlying mandrel providing the overall figure of the mirror. Care must be taken during the lamination and curing process as slight deviations from a symmetric lay-up will cause significant figure errors. Next, the PZT plates are bonded to the backside of the CFRP substrate with the mandrel underneath to provide support (Step 2). A common ground plane 
and patterned electrodes are printed onto either side of the plate before the bonding process takes place. A low viscosity, room-temperature cure epoxy is used for this bonding process in order to ensure even epoxy flow out and to avoid thermal distortions. Once cured, the assembly is removed from the underlying mandrel and bonded to a nanolaminate facesheet (Step 3). This nanolaminate has been deposited on a mandrel with the same figure as that used for the CFRP replication process, producing a matching part. Care must be taken in order to ensure a uniform thickness distribution of epoxy between the CFRP substrate and the nanolaminate during this bonding process. The Kapton electrode routing layer is then incorporated onto the backside of the mirror using conductive epoxy (Step 5) and lastly, the completed CSM is the parted from the secondary mandrel and trimmed to size (Step 6).

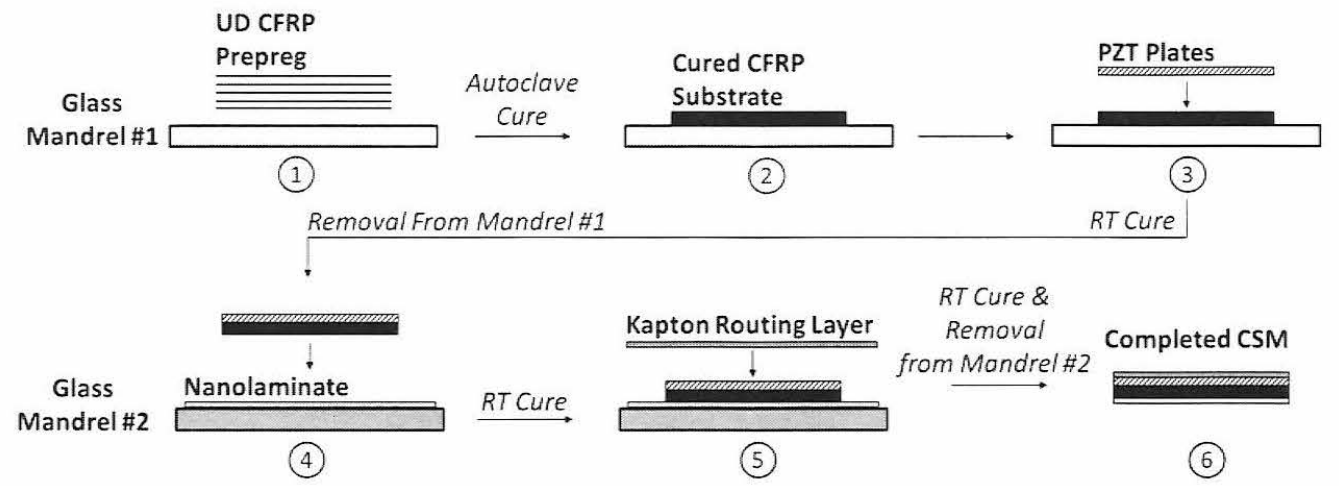

Figure 11. Fabrication schematic for the CSM prototypes.

Figure 12 displays the front and back surface of CSM prototypes constructed using these techniques. These prototypes range from $150-175 \mathrm{~mm}$ in diameter, $500-850 \mu \mathrm{m}$ in total thickness and have an areal density of $1.5-2.7 \mathrm{~kg} / \mathrm{m}^{2}$. They display good surface quality (2.2 $\mathrm{nm}$ Ra surface roughness) and have large actuation capabilities (up to $150 \mu \mathrm{m}$ PV measured influence functions).

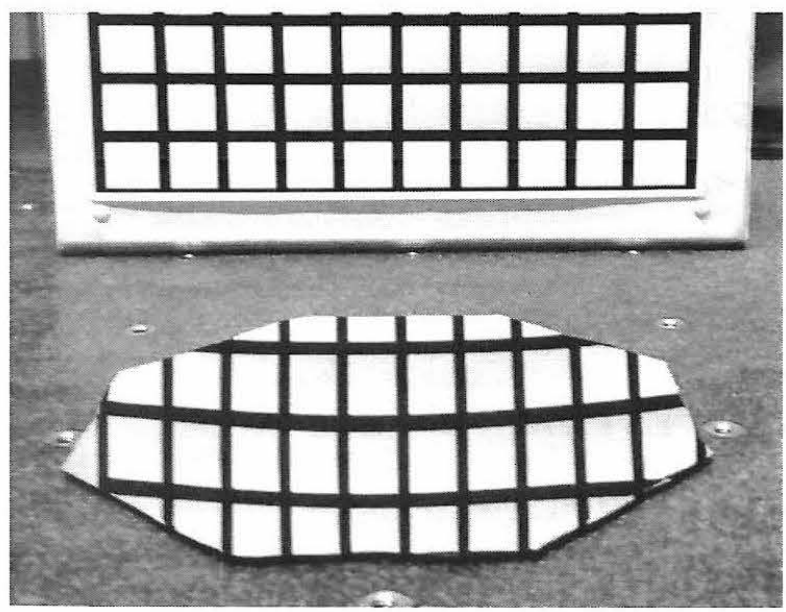

(a)

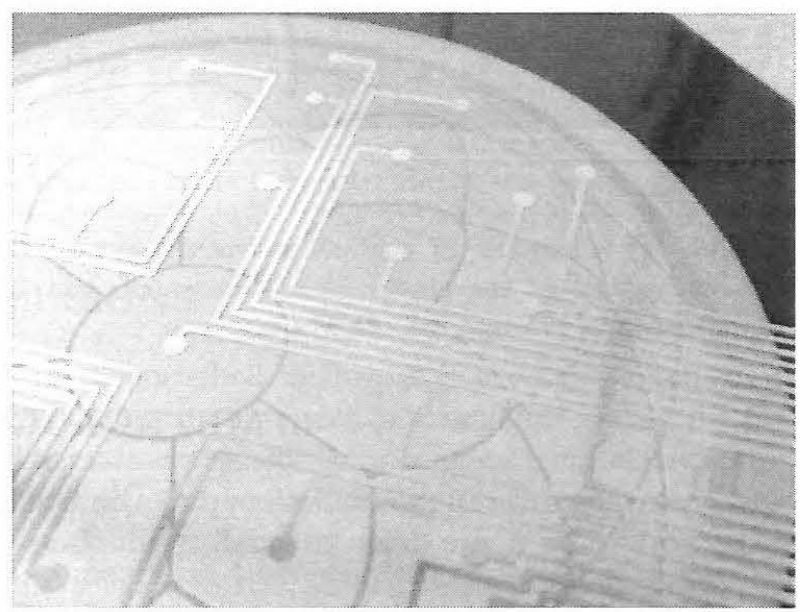

(b)

Figure 12. A CSM prototype displaying (a) the reflective front surface obtained through the nanolaminate integration and (b) the electrode pattern and Kapton routing layer. 


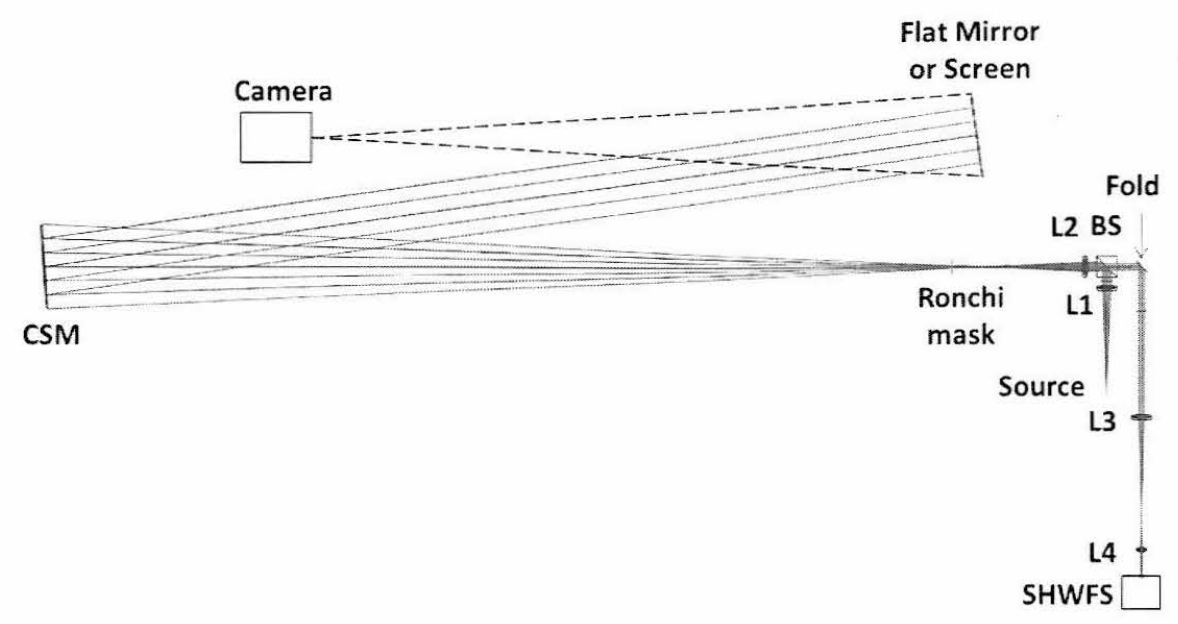

(a)

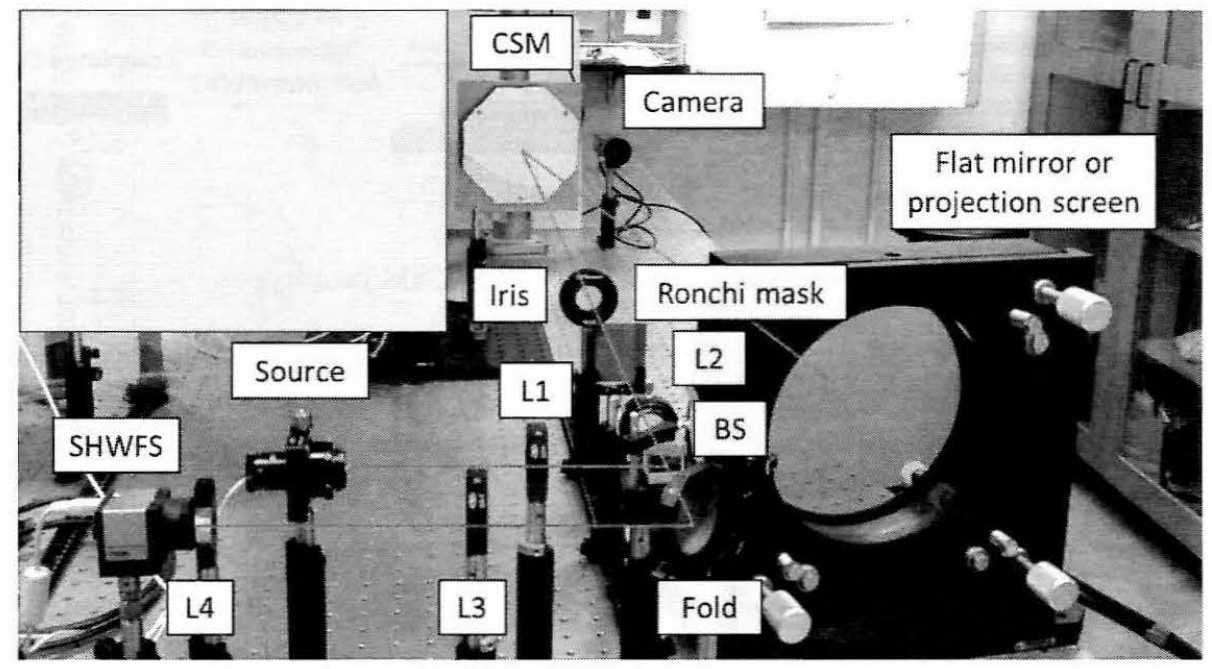

(b)

Figure 13. (a) Schematic of custom metrology setup incorporating the Projected Hartmann test for coarse measurements as well as the classic Shack-Hartmann test to be used for fine corrections. (b) Lab implementation accommodating a $175 \mathrm{~mm}$ dia. CSM prototype

\section{METROLOGY \& TESTING}

It has been shown through simulation that the CSMs can potentially exhibit large actuation capabilities and can correct its figure down to small residual errors. However, the relatively large magnitude of initial shape errors present after manufacturing make it difficult to perform measurements of the initial surface using standard techniques. Therefore, a custom two-stage metrology system with a high-dynamic range was developed. Figure 13 is a schematic of the metrology system as well as a picture of the actual lab implementation. The first stage of the system is a variant of a Projected Hartmann test ${ }^{23}$ where the CSM is illuminated by a regular grid pattern produced by a Ronchi mask. This pattern is reflected off of the CSM and onto a flat screen, where it is imaged using an external camera. The reflected pattern of the sub apertures on this screen is then compared to a regular grid in order to determine the local slope errors associated with the CSM. As this method does not require the CSM to form an image, large magnitudes of slope errors can be accommodated. Figure 14(a) displays a grid-displacement measurement of a highly-aberrated mirror 
using the Projected Hartmann setup. The dominating astigmatic error is evident in the pattern of the spot displacements relative to the nominal grid. The influence functions of the CSM can also be measured with this system. Figure 14(b) is a plot of sample influence functions for a preliminary CSM prototype. Using this information, a coarse correction of the mirror can be performed in order to reduce the magnitude of the initial figure error.

Once this coarse correction has been performed and the figure error has been reduced sufficiently, the second stage of the metrology system can be implemented while keeping the voltage map required to perform the initial correction held constant. At this point, the Ronchi mask is removed from the optical path. In addition to this, the projection screen is removed revealing a flat mirror in its place. This mirror relays the incoming light back off of the CSM and into a Shack-Hartmann Wavefront Sensor (SHWFS) where a more classical measurement procedure can be performed. The influence functions are then remeasured with this setup where they are now defined as deviations from the initial voltage map. This now allows for fine corrections to be performed.

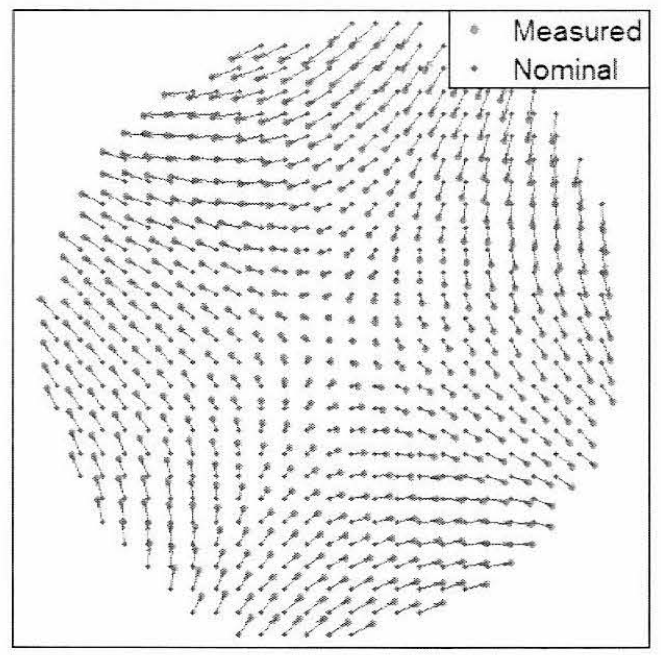

(a)

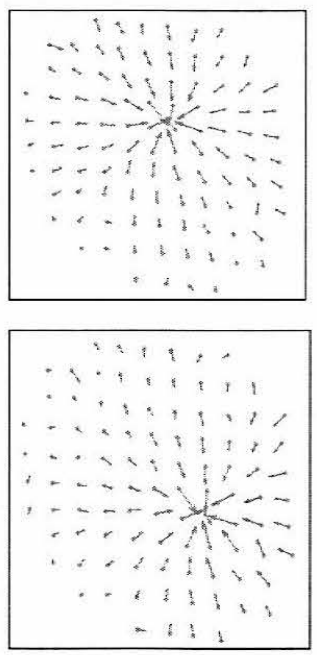

(b)

Figure 14. (a) Projected Hartmann measurement of a CSM prototype showing spot deviations from a regular grid. (b) Sample influence functions from a CSM prototype measured using the Projected Hartmann setup.

\section{CONCLUSIONS}

A novel method of constructing ultra-lightweight active carbon shell mirrors has been presented. These mirrors are laminates of active and passive materials providing the intended mirror figure, reflective frontsurface, actuation capabilities, and electrode wiring. They are extremely thin, ranging from $500-850 \mu \mathrm{m}$ in total thickness with an areal density of $1.5-2.7 \mathrm{~kg} / \mathrm{m}^{2}$; a drastic improvement over traditional mirrors.

A method to size the active layer thickness in order to maximize the actuation capabilities of two CSM designs was presented. With the desire to also minimize the overall mass of the structure and taking into account practical limitations, an active layer thickness of $125 \mu \mathrm{m}$ was chosen as an optimal value both designs. The corrective capability of these two designs with 49 actuator channels was also modeled. It was shown that the 8-ply design had significantly more actuation stroke than the 16-ply design, however both displayed correction factors approaching $99 \%$ before actuator saturation, correcting initial shape errors of up to $50 \mu \mathrm{m}$ down to $<1.0 \mu \mathrm{m}$. Therefore, the CSMs show great potential to correct for large shape errors originating from initial manufacturing errors or thermal deformations. 
Fabrication methods have been developed in order to construct CSM prototypes. They display good surface quality (2.2 $\mathrm{nm}$ Ra surface roughness) and large actuation capabilities (150 $\mu \mathrm{m}$ PV measured influence functions). A custom, two-stage metrology system has also been developed in order to perform both coarse and fine shape corrections of the mirror.

Current efforts are focused on the construction of a fully-integrated 16-ply CSM with a post-correction figure error of $<1.0 \mu \mathrm{m}$ RMS. Several other efforts are underway in order to reduce the magnitude of the initial shape error, reduce the overall thickness of the designs and increase the correction capabilities.

\section{ACKNOWLEDGMENTS}

We thank Keith Patterson, Andrew Shapiro and Scott Basinger (NASA JPL) for their technical and programmatic support throughout the project. Financial support from the Natural Sciences and Engineering Research Council (NSERC) of Canada is gratefully acknowledged. A part of this research was carried out at the Jet Propulsion Laboratory, California Institute of Technology under a contract with the National Aeronautics and Space Administration (NASA).

\section{REFERENCES}

[1] H.P. Stahl, "Design Study of 8 Meter Monolithic Mirror UV/Optical Space Telescope." Society of PhotoOptical Instrumentation Engineers (SPIE) Conference Series 7010 (2008).

[2] J.P. Gardner, J.C. Mather, M. Clampin, et. al. "The James Webb Space Telescope." Space Science Reviews 123.4: 485-606 (2006).

[3] Hochhalter, J. D. "Replicated Mirrors Using Carbon Fiber Reinforced Polymers. Master's Thesis, Univ. of New Mexico (2005).

[4] Chen, P. C., et. al. "Advances in Very Lightweight Composite Mirror Technology." Optical Engineering 39(9) 2320-2329 (2000).

[5] Hickey, G., Barbee, T., Ealey, M., Redding, M. "Actuated Hybrid Mirrors for space telescopes." Society of Photo-Optical Instrumentation Engineers (SPIE) Conference Series 7731 (2010).

[6] Northrup Grumman, AOA Xinetics. http://www.northropgrumman.com/BusinessVentures/AOAXinetics (2014).

[7] Sinquin, J., Lurcon J., Guillemard, C. "Deformable mirror technologies for astronomy at CILAS. Society of Photo-Optical Instrumentation Engineers (SPIE) Conference Series 7015 (2008).

[8] Patterson, K., Pellegrino, S., "Ultralightweight deformable mirrors, Applied Optics 52. pp. 53275341 (2013).

[9] Patterson, K., Pellegrino, S. "Shape correction of thin mirrors in a reconfigurable modular space telescope." Society of Photo-Optical Instrumentation Engineers (SPIE) Conference Series 7731-72 (2010).

[10] Underwood, C., Pellegrino, S. "Autonomous assembly of a reconfigurable space telescope (AAReST) for astronomy and earth observation." 8th IAA Symposium on Small Satellites for Earth Observation, April 4-8, 2011 Berlin, 2011.

[11] Keck Institute for Space Studies - Large Space Structures. http://kiss.caltech.edu/study/largestructure /technology.html (2011).

[12] Postman, M., et al. "The Advanced Technology Large Aperture Space Telescope (ATLAST): Science drivers and technology developments." Optical Engineering 51(1) 011007 (2012).

[13] Planet Labs Inc. http://www.planet.com/ (2014).

[14] Skybox Imaging Inc. http://www.skyboximaging.com/ (2014).

[15] Barbee, T.W. "Nanolaminate thin-shell mirror structures." Society of Photo-Optical Instrumentation Engineers (SPIE) Conference Series 5166 (2003).

[16] Barbee, T.W. "Nano-structure Multilayer Materials", State of the Laboratory, Lawrence Livermore National Laboratory UCRL-5200-91-718 (1991). 
[17] Piezo Systems Inc. http://piezo.com/ (2014).

[18] Fujifilm Inc. Dimatix Materials Printer DMP-2831. http://www.fujifilmusa.com/products/industrial_ inkjet_printheads/deposition-products/dmp-2800/ (2014).

[19] Laslandes, M., Steeves, J., Pellegrino, S., Patterson, K. "Optimization of electrode configuration in surface-parallel actuated deformable mirrors." Society of Photo-Optical Instrumentation Engineers (SPIE) Conference Series 9148-151 (2014).

[20] Jones, R. M., "Mechanics of Composite Materials," 2nd ed. (1998).

[21] SIMULIA. Abaqus Standard/CAE Version 6.12.

[22] Steeves, J., Pellegrino, S. "Ultra-thin highly deformable composite mirrors." 54th AIAA/ASME/ASCE/AHS/ASC Structures, Structural Dynamics and Materials Conference 1523 (2012).

[23] Malacara-Doblado, D., Ghozeil, I., "Hartmann, Hartmann-Shack, and other screen tests. Optical Shop Testing, 3rd ed. Wiley Series in Pure and Applied Optics, pp. 361397 (2007). 